\title{
Chidamide, decitabine, cytarabine, aclarubicin, and granulocyte colony- stimulating factor (CDCAG) in patients with relapsed/refractory acute myeloid leukemia: a single-arm, phase $1 / 2$ study
}

Lixin Wang ${ }^{1,2+}$, Jianmin Luo ${ }^{3+}$, Guofeng Chen ${ }^{4,5,6+}$, Meiyun Fang ${ }^{7}$, Xudong Wei ${ }^{8}$, Yinghua Li ${ }^{9}$, Zhuogang Liu ${ }^{10}$, Yin Zhang ${ }^{11}$, Sujun Gao ${ }^{12}$, Jianliang Shen ${ }^{2}$, Xin Wang ${ }^{13}$, Xiaoning Gao ${ }^{6}$, Wei Zhou ${ }^{5}$, Yigai Ma ${ }^{14}$, Hui Liu ${ }^{15}$, Xinquan $\mathrm{Li}^{16}$, Linhua Yang ${ }^{17}$, Kai Sun ${ }^{11}$ and $\mathrm{Li} \mathrm{Yu}^{1,6^{*}}$ (D)

\begin{abstract}
Background: Epigenetic mechanisms play an important role in the chemoresistance of acute myeloid leukemia (AML). The clinical response to epigenetic modifier-based chemotherapy in patients with relapsed/refractory AML ( $\mathrm{r} /$ $r$ AML) is unclear. This multicenter clinical trial evaluated the safety and efficacy of epigenetic modifiers (chidamide and decitabine) in combination with aclarubicin, cytarabine, and granulocyte colony-stimulating factor (G-CSF) in patients with $r / r$ AML.

Results: Adult patients with r/r AML were treated with chidamide, decitabine, cytarabine, aclarubicin, and G-CSF (CDCAG). The primary measures were overall response (OR), overall survival (OS), and safety. Next-generation sequencing was performed to analyze the correlation between gene mutations and response. A total of 93 patients with $r / r$ AML were enrolled. Overall, 24 patients had a complete remission (CR) and 19 patients achieved CR with incomplete blood count recovery (CRi). The overall response rate (ORR) was $46.2 \%$. The overall survival of these 43 patients who achieved CR/CRi was significantly longer than that of patients who failed to achieve remission (563 vs 152 days, $P<0.0001)$. Of the patients with mutations in epigenetic and transcription factor-related genes, but without internal tandem duplications in FMS-like tyrosine kinase3 (FLT3-ITDs), 55.6\% achieved CR/CRi, whereas the ORR was $28.2 \%$ for patients with mutations in other genes.

(Continued on next page)
\end{abstract}

\footnotetext{
*Correspondence: liyu301@vip.163.com

${ }^{\dagger}$ Lixin Wang, Jianmin Luo and Guofeng Chen contributed equally to this work.

'Department of Hematology-Oncology, International Cancer Center,

Shenzhen University General Hospital, Shenzhen University Health Science Center, Shenzhen, China

${ }^{6}$ Department of Hematology, Chinese PLA General Hospital, 28 Fuxing Road, Beijing 100853, China

Full list of author information is available at the end of the article
}

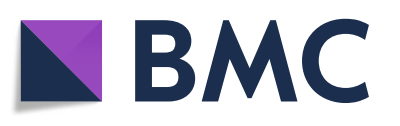

(- The Author(s). 2020 Open Access This article is licensed under a Creative Commons Attribution 4.0 International License, which permits use, sharing, adaptation, distribution and reproduction in any medium or format, as long as you give appropriate credit to the original author(s) and the source, provide a link to the Creative Commons licence, and indicate if changes were made. The images or other third party material in this article are included in the article's Creative Commons licence, unless indicated otherwise in a credit line to the material. If material is not included in the article's Creative Commons licence and your intended use is not permitted by statutory regulation or exceeds the permitted use, you will need to obtain permission directly from the copyright holder. To view a copy of this licence, visit http://creativecommons.org/licenses/by/4.0/. The Creative Commons Public Domain Dedication waiver (http://creativecommons.org/publicdomain/zero/1.0/) applies to the data made available in this article, unless otherwise stated in a credit line to the data. 
(Continued from previous page)

Conclusions: The CDCAG regimen was well tolerated and effective in $r / r$ AML. Patients with epigenetic and transcription factor-related gene mutations, but without FLT3-ITD mutations, may benefit from this regimen.

Trial registration: Clinical Trials, NCT02886559. Registered 01 September 2016

Keywords: Relapsed/refractory acute myeloid leukemia, Next-generation sequencing, Histone deacetylase inhibitor, DNA methyltransferase inhibitor

\section{Introduction}

Approximately $30 \%$ of acute myeloid leukemia (AML) cases will be classified as refractory AML due to failure of induction chemotherapy. Additionally, more than 50\% of patients who achieve complete remission (CR) will eventually relapse [1]. Therefore, the majority of patients with AML will eventually be classified as refractory or relapsed AML (r/r AML). The prognosis for $r / r$ AML remains dismal despite significant effort devoted to the development of novel single-agent drugs and the design of new combination regimens.

Resistance to multiple chemotherapeutic agents is a common clinical problem encountered in $\mathrm{r} / \mathrm{r}$ AML treatment [2]. Accumulating research has demonstrated the importance of epigenetic modification in the pathogenesis of chemoresistance. DNA methylation and histone acetylation are the most common epigenetic changes and can be pharmacologically reversed by DNA methyltransferase (DNMT) inhibitors or histone deacetylase (HDAC) inhibitors. Recent studies have shown that decitabine, a DNMT inhibitor, can increase the chemosensitivity of several leukemic [3] and solid tumor cells [4, 5]. Furthermore, the addition of HDAC inhibitors, such as chidamide or panobinostat, can enhance decitabine's chemosensitization and cytotoxicity effects on leukemia cells when combined with conventional chemotherapy [6-9].

The standard dose of CAG regimen, consisting of lowdose cytarabine, aclarubicin, and granulocyte colonystimulating factor (G-CSF), led to a CR rate of $50 \%$ with well-tolerated toxicity in elderly patients, and the combination with decitabine increased the CR rate to $64.7 \%$ [10-12]. In this regimen, aclarubicin, an anthracycline topoisomerase II inhibitor, was recently reported to induce histone eviction in genomic regions and caused important epigenetic changes $[13,14]$. Therefore, exploring whether the addition of epigenetic modifiers, such as decitabine and further the HDAC inhibitors, to the CAG regimen could exert a clinical benefit in $r / r$ AML patients is of great significance. In this study, we designed a regimen that included chidamide, decitabine, cytarabine, aclarubicin, and G-CSF (the CDCAG regimen) for the treatment of patients with $r / r$ AML. We then conducted a phase I/II study to evaluate the safety and efficacy of this regimen.

\section{Results}

\section{Patient characteristics}

A total of 93 patients, median age 40 years (range, 1860 years), with primary refractory AML $(n=37,39.8 \%)$, early relapsed AML $(n=38,40.9 \%)$, or late relapsed AML ( $n=18,19.4 \%)$ were enrolled in the study. Among the 56 patients with relapsed AML, 40 were experiencing their first relapse, 15 were in their second relapse, and one was experiencing a third relapse. Patients with relapsed AML had a median remission duration before relapse of 7.6 months (range, 1-51 months). Sixty-nine $(74.2 \%)$ patients had received at least three prior treatment regimens, $52(55.9 \%)$ patients had received at least five prior treatment regimens, and 36 (38.7\%) patients had received at least seven prior treatment regimens. Seventeen (18.3\%) patients had received at least one hypomethylating agent, and two patients $(2.2 \%)$ had previously undergone allogeneic hematopoietic stem cell transplantation (allo-HSCT). Patients had received a median of five (1-17) therapeutic cycles prior to enrollment in this study. Patients were enrolled at a median of 8.8 months (1.3-63.8 months) after initial diagnosis. Patient baseline characteristics are summarized in Table 1.

\section{Clinical responses}

Overall, 24/93 (25.8\%) patients achieved CR according to the International Working Group (IWG) criteria and an additional 19/93 (20.4\%) patients achieved CR with incomplete blood count recovery (CRi), with an overall response rate (ORR) of $46.2 \%$ (Table 2). Among the 43 patients who achieved CR/CRi, the median duration of leukemia-free survival was 259 days [ $95 \%$ confidence interval (CI) 215-not available]. Of the 17 patients who received prior hypomethylating agents, nine (52.9\%) achieved CR/CRi.

The median overall survival (OS) was 266 days (95\% CI 235-398 days) with an estimated 6-month OS rate of $67.2 \%$ (95\% CI $57.8-78.0 \%$ ) and a 1 -year OS of $36.9 \%$ (95\% CI 26.6-51.1\%, Fig. 1a). For the 43 patients who achieved CR/CRi, the 6-month relapse-free survival (RFS) rate was $66.2 \%$ (95\% CI 52.7-83.2\%), the 1-year RFS rate was $40.7 \%$ (95\% CI 26.0-63.9\%), and the median RFS was 259 days (95\% CI 185-NA; Fig. 1b). Patients achieving CR/CRi showed a significant improvement in median OS compared with that of non-responders (563 vs 152 days, $P$ 
Table 1 Patient demographics and baseline characteristics $(N=93)$

\begin{tabular}{ll}
\hline Characteristic & Value $^{\$}$ \\
\hline Age, years & $40.0 \pm 12.4$ \\
Sex, no. (\%) & \\
Male & $50(53.8)$ \\
Female & $43(46.2)$ \\
BM blasts, \% & $0.4 \pm 0.3$ \\
HB, g/dL & $91.0 \pm 25.3$ \\
WBC, $\times 10^{9} /$ L & $9.2 \pm 15.9$ \\
PLT, $\times 10^{9} /$ L & $69.5 \pm 66.4$ \\
ECOG PS, no. (\%) & \\
0 & $34(36.6)$ \\
1 & $42(45.2)$ \\
2 & $16(17.2)$ \\
3 & $1(1.1)$ \\
FAB classification, no. (\%) & \\
M0 & $2(2.2)$ \\
M1 & $3(3.2)$ \\
M2 & $49(52.7)$ \\
M4 & $14(15.1)$ \\
M5 & $24(25.8)$ \\
M6 & $1(1.1)$ \\
Dagnis, &
\end{tabular}

Diagnosis, no. (\%)

Refractory

Early relapse

Late relapse

Antecedent hematologic disorders, no. (\%)

$$
\begin{aligned}
& \text { Myelodysplastic syndromes } \\
& \text { Aplastic anemia } \\
& \text { Prior therapies, no. (\%) } \\
& 0-5 \\
& 6-10 \\
& \geq 11
\end{aligned}
$$

Prior therapy, no. (\%)

Prior epigenetic agents

Prior allogeneic stem cell transplant

Karyotype, no. (\%)

Normal karyotype

Complex karyotype

$\mathrm{T}(8 ; 21)$

Mutation counts, no. $(\%)^{*}$

0

$12(13.6)$

$20(22.7)$

$22(25.0)$

19 (21.6)
Table 1 Patient demographics and baseline characteristics $(N=93)$ (Continued)

\begin{tabular}{ll}
\hline Characteristic & Value $^{\text {\$ }}$ \\
\hline 4 & $12(13.6)$ \\
5 & $3(3.4)$
\end{tabular}

Abbreviations: $B M$ bone marrow, $H B$ hemoglobin, $W B C$ white blood cell count, PLT platelets, ECOG PS Eastern Cooperative Oncology Group performance score, FAB French-American-British, CEBPA-dm CEPBA double mutation Descriptive statistics were presented as the mean \pm standard deviation (mean \pm SD) for continuous data and as numbers and percentages for dichotomous/categorical data

* Measured in 88 patients who underwent gene mutation detection

\#Complex karyotype was defined as $\geq 3$ clonal chromosomal abnormalities

$<0.0001$; Fig. 1c). However, the differences of ORR and OS among patients with primary refractory AML, those in their first relapse, and those who had experienced multiple relapses were not statistically significant (data not shown).

Of the 43 patients who achieved CR/CRi, eight patients underwent allo-HSCT treatment. Among these eight patients, the death rate was $12.5 \%$, whereas for the remaining 35 patients who received other treatments, the death rate was $42.9 \%$. Moreover, patients who underwent allo-HSCT showed a trend of longer OS than those received other treatments, although it was not statistically significant $(P=0.0546$; Fig. S1).

\section{Molecular features and patient characteristics}

In total, 88/93 patients underwent gene mutation detection with next-generation sequencing (NGS). Two hundred nine mutations in 184 genes were detected in 76 patients with a median of two mutated genes per patient (range, 0-5). There were 10 mutations found in one patient, seven mutations found in two patients, five mutations in four patients, two mutations in five patients, and 12 mutations in more than five patients. As shown in Fig. 2a and Table S1, the most frequently mutated genes were FLT3-ITD (19.3\%), followed by CEBPA (19.3\%, including $9.7 \%$ with a CEBPA-double mutation [CEBPA$\mathrm{dm}])$, WT1 (18.2\%), RUNX1 (15.9\%), and DNMT3A (13.6\%). No significant differences in the number of mutated genes and their variant allele fractions (VAFs) were found among those experiencing refractory AML, first

Table 2 Best response after 1-2 cycles of the CDCAG therapy ( $N$ =93)

Clinical response $\quad N(\%)$

CR $24(25.8)$

$19(20.4)$
$\mathrm{PR}$ 


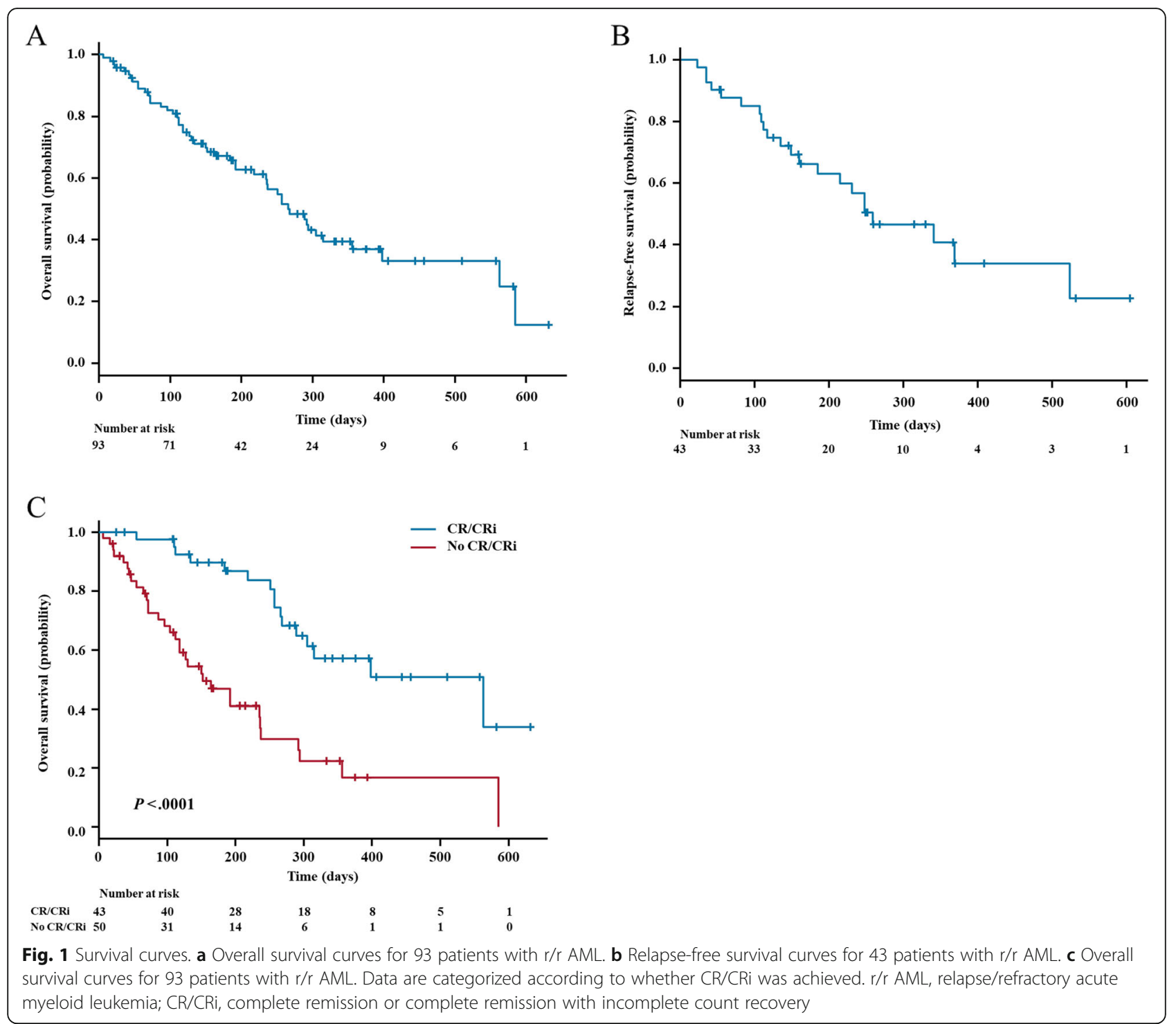

AML relapse, and second or more relapse AML (data not shown).

Among 76 patients with detectable mutations, 20 patients had one mutated gene, 22 patients had two mutated genes, 19 patients had three mutated genes, 11 patients had four mutated genes, and four patients had five mutated genes. In total, $73.7 \%$ (56/76) of the patients harbored co-mutations. The most common comutations were CEBPA with WT1, NPM1 with FLT3ITD, DNMT3A with FLT3-ITD, and DNMT3A with NPM1 (Fig 2b). Among the 88 patients who underwent gene mutation detection, 12 patients had no detectable mutation. No significant differences in age, sex, bone marrow (BM) blasts, white blood cell (WBC), and karyotype were found between the patients with detectable mutations and those without (Table S2).
Correlations between mutation profiles and responses Among the 76 patients who had detectable gene mutations, $33(43.4 \%)$ showed a response (CR/CRi), while 8/ $12(66.7 \%)$ without detectable mutation showed a response. Patients with mutations in IDH2, TET2, GATA2, RAD21, and DNMT3A had ORRs of up to $80.0 \%, 75.0 \%$, $66.7 \%, 66.7 \%$, and $58.3 \%$, respectively, while patients with FLT3-ITD had an ORR of 29.4\% (Table S3 and Fig. S2). However, none of the mutated genes, as well as the comutations, were statistically associated with a better ORR in univariate models (Table S4 and Fig. S3).

Interestingly, the mutation profile differed between patients with a response and those without. To further analyze the correlation between gene mutation and clinical response, we categorized the genes into different functional groups, including DNA methylation-related 


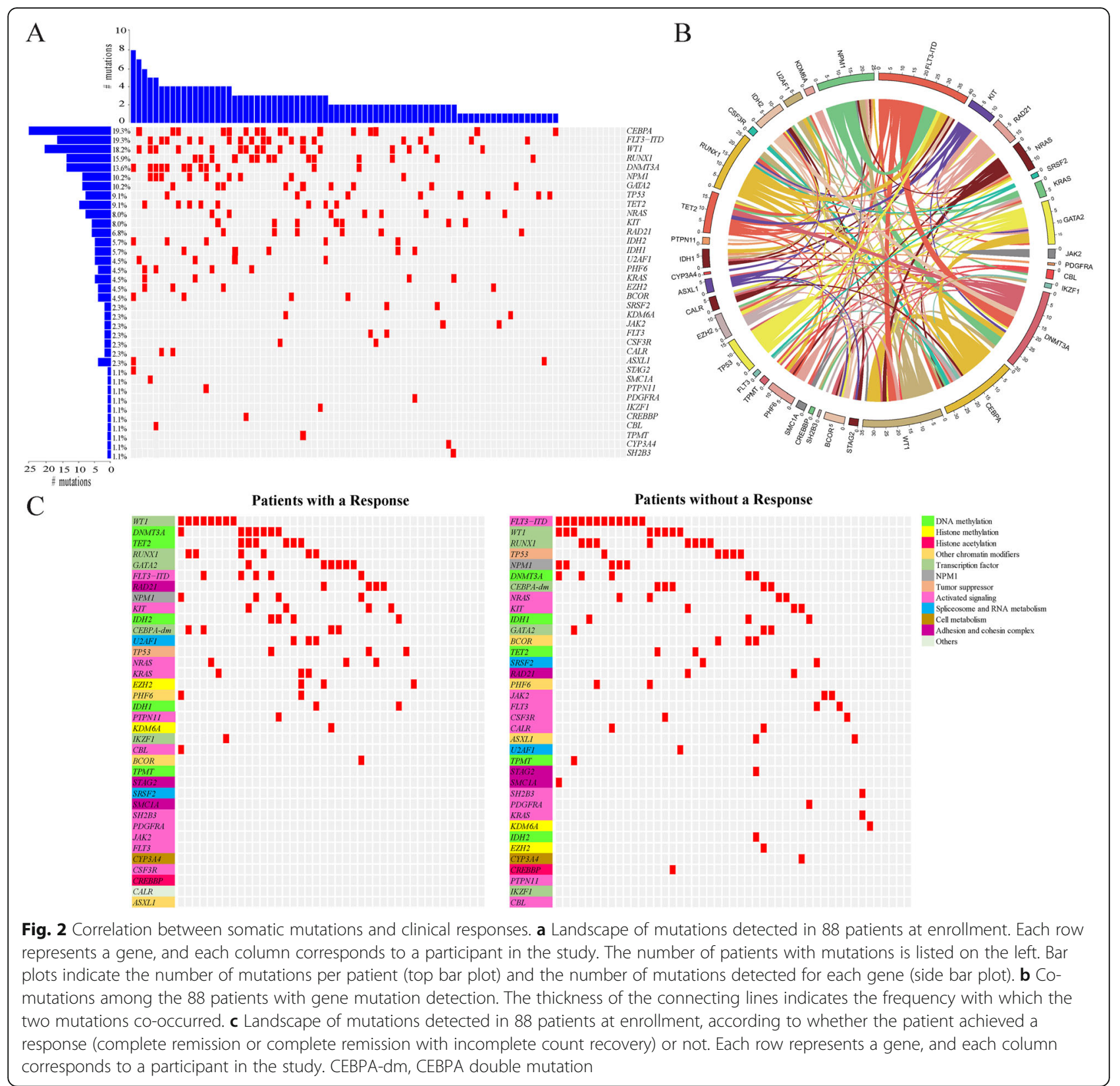

gene mutations (found in $6.8 \%$ of patients), histone methylation-related gene mutations (4.5\%), histone acetylation-related gene mutations $(2.3 \%)$, transcription factor-related gene mutations (18.2\%), and activated signaling-related gene mutations (44.3\%) [15-17] (Table S5). As shown in Fig. 2c, patients who achieved CR/CRi showed more epigenetic modifier-related or transcription factor-related gene mutations but less FLT3-ITD mutations. We then defined the panel, including epigenetic modifier-related or transcription factor-related genes, but without FLT3-ITD co-mutation, as panel ET.
The baseline characteristics were comparable between patients in the panel ET group and those with other mutations (Table S6). We observed that $22 / 37$ (59.5\%) patients in the panel ET group and 11/39 (28.2\%) patients with other mutations achieved CR/CRi $(P=0.006)$, and the difference in ORR was also significant in the multivariate analyses (odds ratio $=4.45, P=0.0085$; Table 3 ). Compared with patients with other mutations, patients in the panel ET group had a better OS $(P=0.0460$; Fig. 3$)$. Moreover, when we excluded the FLT3-ITD mutation, patients in the panel ET group still had a better ORR (59.5\% 
Table 3 Univariate and multivariate models of ORR for patients categorized according to their mutations ( $N=76$ (measured in 76 patients who had detectable gene mutations))

\begin{tabular}{|c|c|c|c|c|}
\hline & \multicolumn{2}{|l|}{ Univariate models } & \multicolumn{2}{|c|}{ Multivariate models } \\
\hline & OR $(95 \% \mathrm{Cl})$ & $P$ value & OR $(95 \% \mathrm{Cl})$ & $P$ value \\
\hline \multicolumn{5}{|l|}{ Category } \\
\hline Other mutations & 1.0 & & 1.0 & \\
\hline Mutations in panel $\mathrm{ET}^{\#}$ & $3.73(1.43,9.73)$ & 0.0070 & $4.45(1.46,13.50)$ & 0.0085 \\
\hline Age & $1.03(0.99,1.07)$ & 0.0920 & $1.04(0.99,1.08)$ & 0.1351 \\
\hline \multicolumn{5}{|l|}{ Sex } \\
\hline Male & 1.0 & & 1.0 & \\
\hline Female & $0.43(0.17,1.11)$ & 0.0823 & $0.39(0.13,1.21)$ & 0.1035 \\
\hline BM blasts & $0.13(0.02,0.80)$ & 0.0275 & $0.32(0.04,3.01)$ & 0.3218 \\
\hline HB & $1.02(1.00,1.04)$ & 0.0244 & $1.01(0.99,1.04)$ & 0.2979 \\
\hline WBC & $1.00(0.98,1.03)$ & 0.9117 & & \\
\hline PLT & $1.01(1.00,1.01)$ & 0.1566 & & \\
\hline \multicolumn{5}{|l|}{ ECOG PS } \\
\hline 0 & 1.0 & & & \\
\hline 1 & $0.24(0.08,0.78)$ & 0.0169 & & \\
\hline 2 & $0.73(0.19,2.90)$ & 0.6580 & & \\
\hline 3 & $0.00(0.00, \ln f)$ & 0.9913 & & \\
\hline \multicolumn{5}{|l|}{ Diagnosis } \\
\hline Refractory & 1.0 & & 1.0 & \\
\hline Early relapse & $2.00(0.69,5.78)$ & 0.2002 & $1.81(0.51,6.47)$ & 0.3600 \\
\hline Late relapse & $4.27(1.09,16.83)$ & 0.0377 & $4.91(0.90,26.80)$ & 0.0663 \\
\hline \multicolumn{5}{|l|}{ Prior therapies } \\
\hline $0-5$ & 1.0 & & & \\
\hline $6-10$ & $1.32(0.48,3.66)$ & 0.5912 & & \\
\hline$\geq 11$ & $1.87(0.49,7.18)$ & 0.3588 & & \\
\hline
\end{tabular}

Abbreviations: $O R$ odds ratio, $C l$ confidence interval, $B M$ bone marrow, $H B$ hemoglobin, WBC white blood cell count, $P L T$ platelets, ECOG PS Eastern Cooperative Oncology Group performance score

\#Panel ET: epigenetic modifier-related or transcription factor-related gene mutations, but without FLT3-ITD co-mutation

vs $27.3 \%, P=0.0170)$ than those with other mutations, although there was no difference in OS $(P=0.13)$.

\section{Safety}

The CDCAG regimen was generally well tolerated in patients with r/r AML. Adverse events (AEs) were reported for all patients and are summarized in Table 4. The most common non-hematologic AEs were infection (including pneumonia), nausea, fatigue, vomiting, hypokalemia, hypoalbuminemia, and febrile neutropenia, the majority of which were grade $1 / 2$. The most common grade $3 / 4$ AEs were infection (including pneumonia) and febrile neutropenia. Serious AEs occurred in 12 (13\%) patients, primarily due to sepsis $(n=3)$, pneumonia $(n=3)$, and cerebral hemorrhage $(n=2)$. There were no instances of tumor lysis syndrome. In addition, four (4.3\%) patients experienced early death (death within 28 days of the start of treatment) due to cerebral hemorrhage $(n=2)$ and sepsis $(n=2)$; only one patient died of disease progression during consolidation.

\section{Discussion}

Globally, the outcomes of patients with $\mathrm{r} / \mathrm{r}$ AML are poor. The chance of achieving $\mathrm{CR}$ with commonly used salvage regimens, including high-dose cytarabine, is less than $15 \%$, and the 1 -year OS rate is less than $10 \%[18$, 19]. In this prospective multicenter trial, $39.8 \%$ of the patients were diagnosed with primary refractory AML, and $67.9 \%$ of patients with relapsed disease were classified as early relapsed AML. The patients were treated with the CDCAG regimen. The CR/CRi rate was $46.2 \%$ and the median OS was 266 days, with a 1 -year OS rate of $36.9 \%$. Thus, the clinical results are encouraging, although it is difficult to compare our study to other trials directly because of the heterogeneity in the population. Notably, NGS was performed on 88 patients in order to 


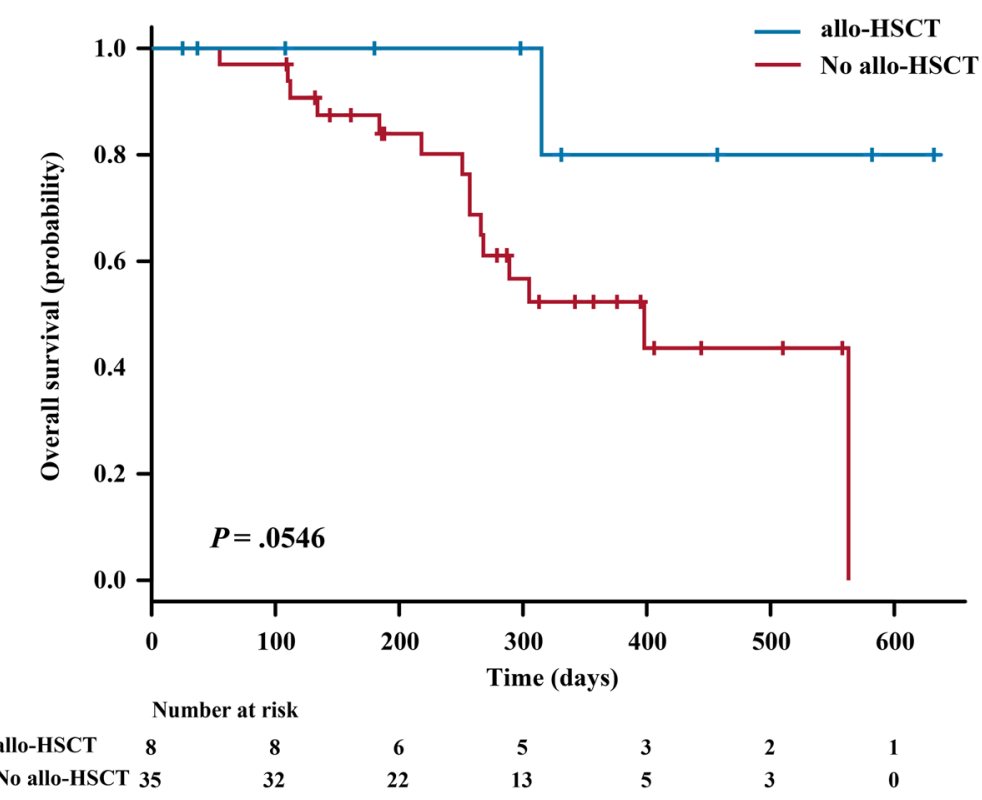

Fig. 3 Overall survival curves for 76 patients with detectable mutation. Data are categorized according to whether mutation was in panel ET*. *Panel ET: epigenetic modifier-related or transcription factor-related gene mutations, but without FLT3-ITD co-mutation

explore the prognosis-associated gene mutation profile. Patients with epigenetic or transcription factor-related gene mutations, but without FLT3-ITD mutations, achieved better responses, which indicated that patients with $\mathrm{r} / \mathrm{r}$ AML may benefit from our regimen.

Table 4 Treatment-related non-hematologic adverse events

\begin{tabular}{lll}
\hline AE* $^{*}$ & Any grade AE, N (\%) & Grade $3 / 4 \mathrm{AE}, N(\%)$ \\
\hline Any AE & $93(100.0)$ & $59(63.4)$ \\
Infection** & $43(46.2)$ & $25(26.9)$ \\
Nausea & $41(44.1)$ & $0(0.0)$ \\
Fatigue & $31(33.3)$ & $1(1.1)$ \\
Vomiting & $31(33.3)$ & $1(1.1)$ \\
Hypokalemia & $29(31.2)$ & $9(9.7)$ \\
Hypoalbuminemia & $25(26.9)$ & $0(0.0)$ \\
Febrile neutropenia & $23(24.7)$ & $23(24.8)$ \\
Pneumonia & $21(22.6)$ & $13(14.0)$ \\
Hypocalcemia & $18(19.4)$ & $1(1.1)$ \\
Cough & $17(18.3)$ & $0(0.0)$ \\
Hyponatremia & $16(17.2)$ & $2(2.2)$ \\
Abdominal pain & $14(15.1)$ & $0(0.0)$ \\
Pharyngalgia & $14(15.1)$ & $1(1.1)$ \\
Anorexia & $12(12.9)$ & $2(2.2)$ \\
Diarrhea & $10(10.8)$ & $0(0.0)$
\end{tabular}

Abbreviations: $A E$ adverse event

${ }^{*}$ AEs were assessed based on the CTCAE (NCI Common Terminology Criteria for Adverse Events) version 5.0 and are shown with a frequency $\geq 10 \%$

**Infection included pneumonia
Chemoresistance is the greatest challenge to be conquered for $\mathrm{r} / \mathrm{r}$ AML patients. In this study, 69 (74.2\%) patients had received at least three cycles of chemotherapy before enrollment, suggesting that leukemic cells in most patients were chemoresistant. In a recent study, cytosine methylation sequencing of genetically diverse AML patients revealed that many of the common AML driver mutations were epigenetic modifiers [20]. Furthermore, a direct epigenetic mechanism for AML chemoresistance was discovered by another study [21]. These studies suggest that the targeting of epigenetic modifiers may represent a new approach to overcome the chemoresistance of $r / r$ AML cells. Decitabine is the first DNMT inhibiter that was used as an induction or salvage therapy to manage AML patients [22-24]. As a single agent, decitabine treatment achieved a $\mathrm{CR}$ rate of 15-21\% in patients with $\mathrm{r} / \mathrm{r}$ AML [24, 25]. Preclinical studies have shown that hypomethylating agents exhibit synergistic activity in leukemia cells when combined with an HDAC inhibitor [9, 26, 27]. Several clinical trials have explored the clinical benefit of a DNMT inhibitor in combination with an HDAC inhibitor for AML patients $[28,29]$. The results of these studies were unsatisfactory since the CR/CRi rate was lower than $15 \%$. In one study, decitabine was given for priming before cytotoxic agents mitoxantrone, etoposide, and cytarabine [30]. While $33 \%$ of patients with $\mathrm{r} / \mathrm{r}$ AML achieved CR/ CRi, seven patients $(15 \%)$ died within 28 days of treatment initiation. In our trial, two classic epigenetic modifiers were included in the regimen: decitabine and chidamide. Chidamide is reported to act synergistically 
with DNA-damaging agents (daunorubicin, idarubicin, and cytarabine) or decitabine to diminish tumor burden in patients with $\mathrm{r} / \mathrm{r}$ AML $[6,31]$. Recently, aclarubicin, an anthracycline topoisomerase II inhibitor, was shown to preferentially induce histone eviction in genomic regions characterized by specific epigenetic modifications [13]. Therefore, three of the agents in our regimen act through epigenetic mechanisms. The results were consistent with our expectations, suggesting that combining epigenetic modifiers with cytotoxic agents was a promising direction for $\mathrm{r} / \mathrm{r}$ AML treatment.

In this study, NGS was performed on 88 patients, which allowed us to identify prognosis-associated gene mutations. For the 12 patients without detectable gene mutations, the ORR was as high as $66.7 \%$. The underlying mechanism is not clear and needs to be clarified in the future. For patients with epigenetic or transcription factor-related gene mutations but without FLT3-ITD, defined as panel ET, the ORR was $59.5 \%$, suggesting that patients with mutations in panel ET should be treated with CDCAG. The finding needs further verification by a prospective, large-scale clinical trial in the future. One study reported that FLT3-ITDs were an independent prognostic factor associated with lower OS among patients with $\mathrm{r} / \mathrm{r}$ AML [32]. Consistent with the study, we found in our trial that patients with FLT3-ITDs had a lower chance of achieving CR. Recently, several tyrosine kinase inhibitors (TKIs, e.g., sorafenib, midostaurin, quizartinib, and crenolanib) have been introduced for the treatment of patients with $\mathrm{r} / \mathrm{r}$ AML with FLT3 mutations (predominantly ITD). This suggests that TKIs may be a suitable addition to our regimen to treat patients with FLT3-ITDs.

Our study was limited in that it followed a traditional single-arm design and did not include a control group. Furthermore, the role of each single agent in this regimen could not be determined.

In conclusion, the CDCAG regimen showed good antileukemic activity and acceptable toxicity. Furthermore, deep sequencing analysis demonstrated that patients with epigenetic or transcription factor-related gene mutations, but without FLT3-ITDs, achieved a better response to this combination chemotherapy. Chemotherapy combining epigenetic modifiers with cytotoxic agents may represent a promising direction for patients with $\mathrm{r} / \mathrm{r}$ AML.

\section{Methods}

\section{Patients and study design}

This single-arm, multicenter, prospective clinical trial evaluated the safety and efficacy of the CDCAG regimen in patients with $\mathrm{r} / \mathrm{r}$ AML (NCT02886559). This study was conducted in accordance with the Declaration of Helsinki and was approved by the Institutional Review
Board at each participating institution. All patients enrolled in the study provided written informed consent.

From June 2016 to June 2018, 93 patients were enrolled at 14 hospitals in China. Adults with r/r AML, defined according to the standard IWG criteria [33], aged between 18 and 60 years, were eligible for this study. Eligible patients must not have received radiotherapy, chemotherapy, targeted therapy, hematopoietic stem cell transplantation, or any other treatment within 4 weeks prior to enrollment. Furthermore, eligible patients were required to have an ECOG performance status [34] $\leq 3$ and an expected survival time $>3$ months. Patients with active infection, bleeding, new thrombosis, and serious heart, lung, liver, or kidney disease were excluded. The full list of inclusion and exclusion criteria is presented in Table S7.

All patients in this study were treated with the CDCAG regimen (Fig. S4) over a 28-day cycle: chidamide (30 mg, twice per week, days 1-14) and decitabine $\left(20 \mathrm{mg} / \mathrm{m}^{2} /\right.$ day, days $\left.1-5\right)$ in combination with cytarabine $\left(50 \mathrm{mg} / \mathrm{m}^{2} /\right.$ day, days $1-7$ if $\mathrm{WBC} \geq 20 \times 10^{9} / \mathrm{L}$ and days $3-7$ if $\left.\mathrm{WBC}<20 \times 10^{9} / \mathrm{L}\right)$, aclarubicin $\left(10 \mathrm{mg} / \mathrm{m}^{2} /\right.$ day, days 3-7), and granulocyte colony-stimulating factor $\left(300 \mu \mathrm{g} /\right.$ day until $\left.\mathrm{WBC}>20 \times 10^{9} / \mathrm{L}\right)$. Supportive treatment, including anti-infection prophylaxis and growth factor support, was allowed at the investigator's discretion. If morphologic $\mathrm{CRi}$ was achieved [33] and WBC $<2 \times 10^{9} / \mathrm{L}$, a subsequent cycle could be delayed by up to 14 days. All patients received two cycles of the CDCAG regimen. Next, hematological response and toxicity were evaluated.

\section{Definitions of events and end points}

Bone marrow aspiration and biopsy were performed upon screening and at day 28 of cycles 1 and 2. Physical exams, clinical laboratory tests, and monitoring of AEs were performed at screening and throughout the study. Response assessments were categorized according to the IWG criteria. The primary end point was treatment success, which was defined as CR or CRi after completion of the CDCAG regimen treatment. The ORR included the CR/CRi rate. AEs were graded according to the National Cancer Institute Common Terminology Criteria for Adverse Events (NCI-CTCAE) version 5.0 (http:// ctep.cancer.gov). The definitions of events and end points are described in detail in Table S8.

\section{Next-generation sequencing and analysis}

Bone marrow aspiration samples for mutational analysis were collected before treatment. Genomic DNA extracted from bone marrow was examined for mutations using the target sequencing panel, which covered the entire coding sequences of 127 genes known to be relevant to AML pathogenesis (Annoroad Gene Technology, 
Table S5). NimbelGen SeqCap EZ Choice was used in accordance with the manufacturer's protocol, with modifications. Multiplexed libraries were sequenced using 75 bp paired-end runs on Illumina Nextseq 550AR.

Each sample was required to have an average effective depth $\geq 1000 \times$ in the target area. Using the BurrowsWheeler Alignment algorithm to compare the sequence data with the human genome (GRCh37), Picard was used to mark the polymerase chain reaction duplicates, and the quality value of the sequence alignment results was corrected by means of BaseRecalibrator in Genome Analysis Toolkit. MuTect2 software was employed for mutation detection, and all testing mutations were annotated by the ANNOVAR software. The types of analysis included single-nucleotide variants (SNVs), insertions, and deletions (INDELs). Somatic mutations were identified through comparison with the COSMIC (v18) and 1000 Genomes cohort databases, while single-nucleotide polymorphisms described in the dbSNP (v135) database were excluded. The VAF cutoff was set to 0.01 for inclusion in the analyses.

\section{Statistical analysis}

The data cutoff for this report was June 30, 2018. Assessments at screening served as baseline data. Descriptive statistics are presented as the mean \pm standard deviations (mean $\pm \mathrm{SD}$ ) for continuous data and as numbers and percentages for dichotomous/categorical data. Chi-square $\left(\chi^{2}\right)$ /Fisher's exact test was used for categorical variables, and the Kruskal-Wallis test was used for continuous variables. Survival functions were estimated using the KaplanMeier method and were compared using the log-rank test. Associations with OS were assessed using a Cox proportional hazards model. Logistic regression was used to examine the associations between these variables and response rates. Variables significant at $P<0.10$ in univariate analyses were entered into an explorative multivariable model. We also adjusted for features that, when added to this model, changed the matched odds ratio by at least 10\%. All analyses were performed using Empower Stats (X\&Y Solutions, Inc., Boston, MA, USA) and R (version 3.3.3). A two-sided $P$ value $<0.05$ was considered statistically significant.

\section{Role of the funding source}

Chinese PLA General Hospital investigators designed the study and performed all data collection, data analysis, data interpretation, and wrote the manuscript. The funder National Natural Science Foundation of China had no role in study design, data collection, data analysis, data interpretation, or writing of the report. All authors had full access to all the data in the study and the corresponding author had final responsibility for the decision to submit for publication.

\section{Supplementary information}

Supplementary information accompanies this paper at https://doi.org/10. 1186/s13148-020-00923-4.

Additional file 1: Table S1. Gene mutations of patients with $r / r$ AML (N $=88^{*}$ ). Table S2. Baseline characteristics for patients undergoing gene mutation detection ( $N=88$ ). Table $\mathbf{S 3}$. Clinical responses of patients with different mutations. Table S4. Univariate models of overall response rate in patients with different mutations. Table S5. Next-generation sequencing of 127-gene mutation panel in r/r AML (Annoroad Gene Technology). Table S6. Baseline characteristics and outcomes for patients with detectable mutations ( $N=76$ ). Table S7. Study inclusion and exclusion criteria. Table S8. Definitions of events and end points [1]. Fig. S1 Overall survival curves for 43 patients who achieved CR/CRi. Data are categorized according to whether the patient underwent allo-HSCT. Abbreviations: allo-HSCT, allogeneic hematopoietic stem cell transplantation. Fig. S2 The overall response rate of patients with indicated mutations. Abbreviations: $C R$, complete remission; $C R i$, complete remission with incomplete blood count recovery; CEBPA-dm, CEBPA double mutation. Fig. S3 Percentage of patients who achieved and did not achieve CR/CRi among the 88 patients who underwent gene mutation detection. Abbreviations: $C R$, complete remission; $C R i$, complete remission with incomplete blood count recovery; CEBPA-dm, CEBPA double mutation. Fig. S4 Study design. Abbreviations: WBC, white blood cell count; G-CSF, Granulocyte colony-stimulating factor.

\section{Acknowledgements}

The authors thank the participating investigators and patients and thank the NATIVE EE Company for English editing.

\section{Authors' contributions}

Provided funding support: LY. Conceived and designed the experiments: LXW and LY. Performed the experiments: LXW, JML, GFC, MYF, XDW, YHL, $Z G L, Y Z, S J G, J L S, X W, X N G, W Z, Y G M, H L, X Q L, L H Y, K S$, and LY. Analyzed the data: $L X W, J M L, G F C$, and LY. Wrote the paper: $L X W, G F C$, and LY. All authors read and approved the final manuscript.

\section{Funding}

This work was supported by the National Natural Science Foundation of China $(81670162,81470010,81350004)$. The funders had no role in the study design, data collection and analysis, decision to publish, or preparation of the manuscript.

\section{Availability of data and materials}

The data that support the findings of this study are available from the Chinese PLA General Hospital, but restrictions apply to the availability of these data, which were used under license for the current study, and, thus, are not publicly available. However, data are available from the authors upon reasonable request and with permission of the Chinese PLA General Hospital.

\section{Ethics approval and consent to participate}

This single-arm, multicenter, prospective clinical trial evaluated the safety and efficacy of the CDCAG regimen in patients with $r / r$ AML (NCT02886559). This study was conducted in accordance with the Declaration of Helsinki and was approved by the Institutional Review Board at each participating institution. All patients enrolled in the study provided written informed consent.

Consent for publication

Not applicable.

\section{Competing interests}

The authors declare that they have no competing interests.

\section{Author details}

'Department of Hematology-Oncology, International Cancer Center, Shenzhen University General Hospital, Shenzhen University Health Science Center, Shenzhen, China. ${ }^{2}$ Department of Hematology, The Sixth Medical Center, Chinese General Hospital of PLA, Beijing, China. ${ }^{3}$ Department of Hematology, The Second Hospital of Hebei Medical University, Shijiazhuang, 
China. ${ }^{4}$ Department of Endoscopy, Tianjin Medical University Cancer Institute and Hospital, National Clinical Research Center for Cancer, Tianjin's Clinical Research Center for Cancer, Key Laboratory of Cancer Prevention and Therapy, Tianjin, China. ${ }^{5}$ School of Medicine, Nankai University, Tianjin, China. ${ }^{6}$ Department of Hematology, Chinese PLA General Hospital, 28 Fuxing Road, Beijing 100853, China. ${ }^{7}$ Department of Hematology, The First Affiliated Hospital of Dalian Medical University, Dalian, China. ${ }^{8}$ Department of Hematology, The Affiliated Cancer Hospital of Zhengzhou University, Henan Cancer Hospital, Zhengzhou, China. ${ }^{9}$ Department of Hematology, The First Affiliated Hospital of Harbin Medical University, Harbin, China. ${ }^{10}$ Department of Hematology, Shengjing Hospital of China Medical University, Shenyang, China. ${ }^{11}$ Department of Hematology, Henan Provincial People's Hospital, Zhengzhou, China. ${ }^{12}$ Department of Hematology, The First Hospital, Jilin University, Changchun, China. ${ }^{13}$ Department of Hematology, Shandong Provincial Hospital Affiliated to Shandong University, Jinan, China.

${ }^{14}$ Department of Hematology, ChinaJapan Friendship Hospital, Beijing, China. ${ }^{15}$ Department of Hematology, Beijing Hospital, National Center of Gerontology, Beijing, China. ${ }^{16}$ Department of Hematology, Beijing Tsinghua Changgung Hospital, Tsinghua University, Beijing, China. ${ }^{17}$ Department of Hematology, The Second Hospital of Shanxi Medical University, Taiyuan, China.

Received: 22 April 2020 Accepted: 19 August 2020

Published online: 01 September 2020

\section{References}

1. Dohner H, Estey E, Grimwade D, Amadori S, Appelbaum FR, Buchner T, et al. Diagnosis and management of AML in adults: 2017 ELN recommendations from an international expert panel. Blood. 2017;129:424-47.

2. Shaffer BC, Gillet JP, Patel C, Baer MR, Bates SE, Gottesman MM. Drug resistance: still a daunting challenge to the successful treatment of AML. Drug Resist Updat. 2012;15:62-9.

3. Bhatla T, Wang J, Morrison DJ, Raetz EA, Burke MJ, Brown P, et al. Epigenetic reprogramming reverses the relapse-specific gene expression signature and restores chemosensitivity in childhood B-lymphoblastic leukemia. Blood. 2012;119:5201-10.

4. Iwata $H$, Sato $H$, Suzuki $R$, Yamada R, Ichinomiya $S$, Yanagihara $M$, et al. A demethylating agent enhances chemosensitivity to vinblastine in a xenograft model of renal cell carcinoma. Int J Oncol. 2011;38:1653-61.

5. Mirza S, Sharma G, Pandya P, Ralhan R. Demethylating agent 5-aza-2deoxycytidine enhances susceptibility of breast cancer cells to anticancer agents. Mol Cell Biochem. 2010;342:101-9.

6. Mao J, Li S, Zhao H, Zhu Y, Hong M, Zhu H, et al. Effects of chidamide and its combination with decitabine on proliferation and apoptosis of leukemia cell lines. Am J Transl Res. 2018;10:2567-78.

7. Chen L, Mi RH, Zhu ST, Yu P, Wei XD. Therapeutic effect of chidamide and decitabine in combiantion with CHAG priming regimen for 8 patients with relapsed/refractory acute myeloid leukemia. Zhonghua Xue Ye Xue Za Zhi. 2018:39:602-4

8. Savickiene J, Treigyte G, Borutinskaite W, Navakauskiene R. Antileukemic activity of combined epigenetic agents, DNMT inhibitors zebularine and RG108 with HDAC inhibitors, against promyelocytic leukemia HL-60 cells. Cell Mol Biol Lett. 2012;17:501-25.

9. Blagitko-Dorfs N, Schlosser P, Greve G, Pfeifer D, Meier R, Baude A, et al. Combination treatment of acute myeloid leukemia cells with DNMT and HDAC inhibitors: predominant synergistic gene downregulation associated with gene body demethylation. Leukemia. 2019;33:945-56.

10. Huang J, Hong M, Zhu Y, Zhao H, Zhang X, Wu Y, et al. Decitabine in combination with G-CSF, low-dose cytarabine and aclarubicin is as effective as standard dose chemotherapy in the induction treatment for patients aged from 55 to 69 years old with newly diagnosed acute myeloid leukemia. Leuk Lymphoma. 2018;59:2570-9.

11. Li J, Chen Y, Zhu Y, Zhou J, Xu Y, Li Y, et al. Efficacy and safety of decitabine in combination with G-CSF, low-dose cytarabine and aclarubicin in newly diagnosed elderly patients with acute myeloid leukemia. Oncotarget. 2015; 6:6448-58.

12. Qian SX, Li JY, Tian T, Shen YF, Jiang YQ, Lu H, et al. Effect of low-dose cytarabine and aclarubicin in combination with granulocyte colonystimulating factor priming (CAG regimen) on the outcome of elderly patients with acute myeloid leukemia. Leuk Res. 2007;31:1383-8.
13. Pang B, de Jong J, Qiao X, Wessels LF, Neefjes J. Chemical profiling of the genome with anti-cancer drugs defines target specificities. Nat Chem Biol. 2015;11:472-80.

14. Pang B, Qiao X, Janssen L, Velds A, Groothuis T, Kerkhoven R, et al. Druginduced histone eviction from open chromatin contributes to the chemotherapeutic effects of doxorubicin. Nat Commun. 2013;4:1908.

15. Roy DM, Walsh LA, Chan TA. Driver mutations of cancer epigenomes. Protein Cell. 2014;5:265-96.

16. Cancer Genome Atlas Research N, Ley TJ, Miller C, Ding L, Raphael BJ, Mungall AJ, et al. Genomic and epigenomic landscapes of adult de novo acute myeloid leukemia. N Engl J Med. 2013; 368: 2059-74.

17. Metzeler KH, Herold T, Rothenberg-Thurley M, Amler S, Sauerland MC, Gorlich D, et al. Spectrum and prognostic relevance of driver gene mutations in acute myeloid leukemia. Blood. 2016;128:686-98.

18. Uy GL, Rettig MP, Motabi IH, McFarland K, Trinkaus KM, Hladnik LM, et al. A phase $1 / 2$ study of chemosensitization with the CXCR4 antagonist plerixafor in relapsed or refractory acute myeloid leukemia. Blood. 2012;119:3917-24.

19. Roboz GJ, Rosenblat T, Arellano M, Gobbi M, Altman JK, Montesinos P, et al. International randomized phase III study of elacytarabine versus investigator choice in patients with relapsed/refractory acute myeloid leukemia. J Clin Oncol. 2014;32:1919-26.

20. Glass JL, Hassane D, Wouters BJ, Kunimoto H, Avellino R, Garrett-Bakelman $\mathrm{FE}$, et al. Epigenetic identity in AML depends on disruption of nonpromoter regulatory elements and is affected by antagonistic effects of mutations in epigenetic modifiers. Cancer Discov. 2017;7:868-83.

21. Maganti HB, Jrade H, Cafariello C, Manias Rothberg JL, Porter CJ, YockellLelievre J, et al. Targeting the MTF2-MDM2 axis sensitizes refractory acute myeloid leukemia to chemotherapy. Cancer Discov. 2018;8:1376-89.

22. Welch JS, Petti AA, Miller CA, Fronick CC, O'Laughlin M, Fulton RS, et al. TP53 and decitabine in acute myeloid leukemia and myelodysplastic syndromes. N Engl J Med. 2016:375:2023-36.

23. Kadia TM, Cortes J, Ravandi F, Jabbour E, Konopleva M, Benton CB, et al. Cladribine and low-dose cytarabine alternating with decitabine as front-line therapy for elderly patients with acute myeloid leukaemia: a phase 2 singlearm trial. Lancet Haematol. 2018;5:e411-e21.

24. Ritchie EK, Feldman EJ, Christos PJ, Rohan SD, Lagassa CB, Ippoliti C, et al. Decitabine in patients with newly diagnosed and relapsed acute myeloid leukemia. Leuk Lymphoma. 2013;54:2003-7.

25. Khan N, Hantel A, Knoebel RW, Artz A, Larson RA, Godley LA, et al. Efficacy of single-agent decitabine in relapsed and refractory acute myeloid leukemia. Leuk Lymphoma. 2017;58:1-7.

26. Momparler RL, Idaghdour Y, Marquez VE, Momparler LF. Synergistic antileukemic action of a combination of inhibitors of DNA methylation and histone methylation. Leuk Res. 2012;36:1049-54.

27. Nishioka C, Ikezoe T, Yang J, Udaka K, Yokoyama A. Simultaneous inhibition of DNA methyltransferase and histone deacetylase induces p53independent apoptosis via down-regulation of $\mathrm{Mcl}-1$ in acute myelogenous leukemia cells. Leuk Res. 2011;35:932-9.

28. Kirschbaum M, Gojo I, Goldberg SL, Bredeson C, Kujawski LA, Yang A, et al. A phase 1 clinical trial of vorinostat in combination with decitabine in patients with acute myeloid leukaemia or myelodysplastic syndrome. $\mathrm{Br}$ J Haematol. 2014;167:185-93.

29. Tan P, Wei A, Mithraprabhu S, Cummings N, Liu HB, Perugini M, et al. Dual epigenetic targeting with panobinostat and azacitidine in acute myeloid leukemia and high-risk myelodysplastic syndrome. Blood Cancer J. 2014;4: e170

30. Halpern AB, Othus M, Huebner EM, Buckley SA, Pogosova-Agadjanyan EL, Orlowski KF, et al. Mitoxantrone, etoposide and cytarabine following epigenetic priming with decitabine in adults with relapsed/refractory acute myeloid leukemia or other high-grade myeloid neoplasms: a phase 1/2 study. Leukemia. 2017;31:2560-7.

31. Li Y, Wang Y, Zhou Y, Li J, Chen K, Zhang L, et al. Cooperative effect of chidamide and chemotherapeutic drugs induce apoptosis by DNA damage accumulation and repair defects in acute myeloid leukemia stem and progenitor cells. Clin Epigenetics. 2017;9:83.

32. Chevallier $P$, Labopin $M$, Turlure $P$, Prebet T, Pigneux A, Hunault M, et al. A new Leukemia Prognostic Scoring System for refractory/relapsed adult acute myelogeneous leukaemia patients: a GOELAMS study. Leukemia. 2011; 25:939-44.

33. Cheson BD, Bennett JM, Kopecky KJ, Buchner T, Willman CL, Estey EH, et al. Revised recommendations of the international working group for diagnosis, 
standardization of response criteria, treatment outcomes, and reporting standards for therapeutic trials in acute myeloid leukemia. J Clin Oncol. 2003;21:4642-9.

34. Oken MM, Creech RH, Tormey DC, Horton J, Davis TE, McFadden ET, et al. Toxicity and response criteria of the Eastern Cooperative Oncology Group. Am J Clin Oncol. 1982;5:649-55.

\section{Publisher's Note}

Springer Nature remains neutral with regard to jurisdictional claims in published maps and institutional affiliations.

Ready to submit your research? Choose BMC and benefit from:

- fast, convenient online submission

- thorough peer review by experienced researchers in your field

- rapid publication on acceptance

- support for research data, including large and complex data types

- gold Open Access which fosters wider collaboration and increased citations

- maximum visibility for your research: over $100 \mathrm{M}$ website views per year

At BMC, research is always in progress.

Learn more biomedcentral.com/submissions 